\title{
Interferencias morfosintácticas del quechua al castellano: un estudio en el distrito de Jangas, Áncash
}

\section{Morphosyntactic interference from quechua to spanish: a study in Jangas district, Ancash}

\author{
Cecilia de las Nieves Valverde Chávez \\ Universidad Nacional Mayor de San Marcos, Lima, Perú \\ ceciliavalverdeo7@gmail.com
}

\begin{abstract}
Resumen
El castellano hablado en Jangas es una variedad dialectal resultado del contacto lingüístico entre el castellano estándar del Perú y el quechua hablado en Jangas, distrito de la provincia de Huaraz en el departamento de Áncash. Este contacto de lenguas genera diversos tipos de interferencias lingüísticas, como es el caso de la interferencia fónica, morfológica, sintáctica y léxica. El propósito de este artículo es analizar las interferencias morfosintácticas de la lengua origen (quechua) a la lengua meta (español) en hablantes procedentes de Jangas. Los datos obtenidos en el desarrollo de esta investigación muestran como resultado fenómenos como omisión lingüística, discordancia gramatical, inserción lingüística, pronombres anómalos, dequeísmo, etc. Finalmente, se concluye que el fenómeno con mayor marcadez es el de discordancia gramatical, ello puede ser producto de que este fenómeno es el más común en situaciones de contacto de lenguas.
\end{abstract}

Palabras clave: castellano de Jangas, contacto lingüístico, quechua, interferencia y morfosintaxis.

\begin{abstract}
The Andean Spanish spoken in Jangas is a dialect variety resulting from language contact between the standard Spanish of Peru and the quechua spoken in Jangas, district of the province of Huaraz in the department of Ancash. This contact of languages generates different types of linguistics interferences, such as phonic, morphological, syntactic and lexical interference. The purpose of this article is analyze the morphosyntactic interferences from the source language (quechua) to the target language (Spanish) in speakers from Jangas. The data obtained in the development of this research show as a result phenomena such as linguistic omission, grammatical disagreement, linguistic insertion, anomalous pronouns, dequeism, etc. Finally, it is concluded that the phenomenon with greater markedness is that of grammatical disagreement, this may be the product of this phenomenon being the most common in language contact situations.
\end{abstract}

Keywords: Andean Spanish, language contact, quechua, interference and morphosyntaxis. 


\section{Introducción}

El castellano bilingüe y el castellano andino son variedades del castellano producto del contacto de lenguas amerindias y el castellano. En palabras de Ana María Escobar (1990, p. 190), los hablantes de español bilingüe son hablantes maternos del quechua que hablan el español como segunda lengua, es decir, son bilingües consecutivos de español. En este trabajo, haremos referencia a esta variedad del castellano, ya que los colaboradores adquirieron el español como segunda lengua, siendo su primera lengua el quechua.

Según Alberto Escobar (1978), los hablantes de esta variedad provienen de zonas rurales de la región andina, es decir, hablantes que habitan en los departamentos de la sierra del Perú. Por este motivo, el trabajo fue desarrollado en Jangas, distrito de la provincia de Huaraz en el departamento de Áncash. La figura 1 representa el área de estudio de esta investigación.

Figura 1. Zona de Jangas, distrito de la provincia de Huaraz en el departamento de Áncash.

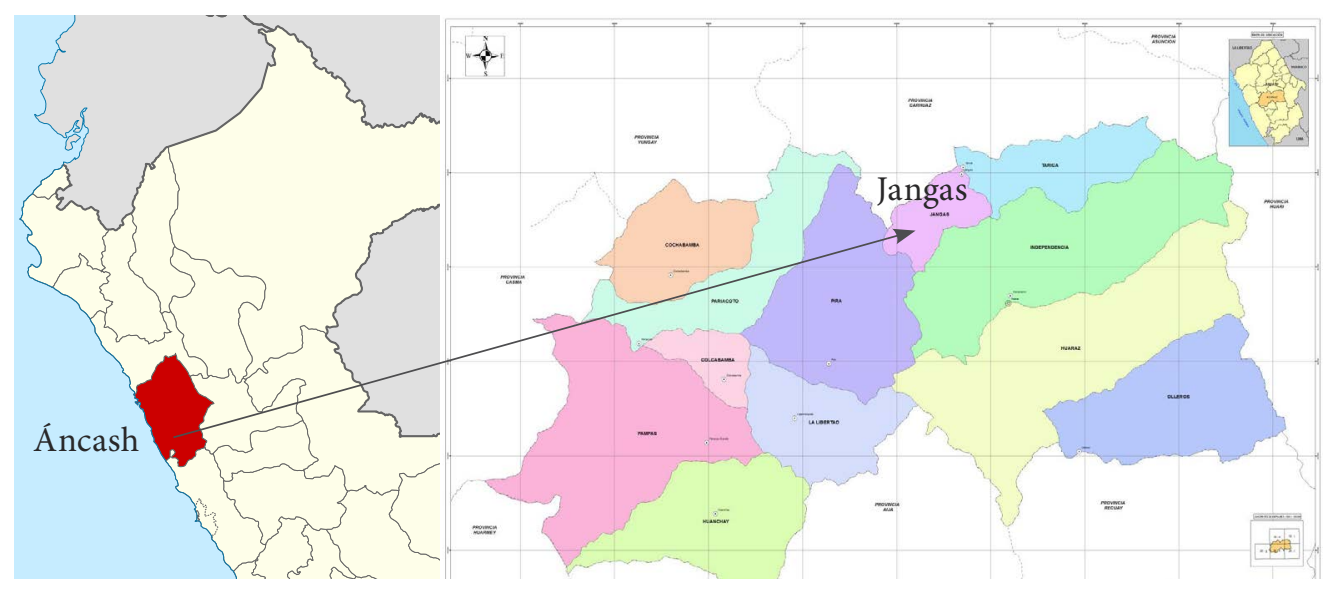

El presente trabajo, enmarcado dentro del nivel morfosintáctico de la lengua, comprende el área del castellano bilingüe propiamente denominado por (Escobar, 1978) hablado por adultos del sexo masculino del distrito de Jangas (Áncash). En esta variedad del castellano, encontramos el empleo recurrente de diversos fenómenos lingüísticos presentados como resultado de la interferencia lingüística de la lengua quechua al español. 
El objetivo que se plantea es analizar las interferencias morfosintácticas de la lengua origen (quechua) a la lengua meta (español) en hablantes procedentes de Jangas. Los datos obtenidos en el desarrollo de esta investigación muestran como resultado fenómenos como omisión lingüística, discordancia gramatical, inserción lingüística, pronombres anómalos, dequeísmo, loísmo, etc. El fenómeno con mayor marcadez es el de discordancia gramatical, ello puede ser producto de que este fenómeno es el más común en situaciones de contacto de lenguas.

Con este propósito, el trabajo se estructura en cinco partes, la primera parte detalla la zona de estudio y los objetivos trazados. La segunda parte abarca la presentación teórica de los conceptos del presente trabajo referidos a las temáticas de castellano bilingüe, contacto de lenguas, interlecto e interferencia lingüística. La tercera, detalla la metodología utilizada para la recolección de los datos. La cuarta parte, referida al análisis, describe y ejemplifica cada interferencia morfosintáctica encontrada en el contexto del castellano bilingüe de Jangas. Como parte final se presentan las conclusiones y la bibliografía utilizada para la elaboración del trabajo.

\section{El contacto de lenguas y el interlecto}

El contacto de lenguas es una situación que ocurre cuando dos o más lenguas son habladas en una misma comunidad. Según Weinreich (1974, p. 17), «dos o más lenguas están en contacto si son usadas alternativamente por las mismas personas, y los individuos que usan las lenguas son, por tanto, el punto de contacto». De igual manera, Silva-Corvalán (1989, p.170), afirma que «dos o más lenguas entran en contacto cuando son usadas por los mismos individuos, es decir, cuando existe una situación de bilingüismo en la que los hablantes bilingües constituyen el locus de contacto». El desarrollo de investigación, gira en torno al resultado de este contacto lingüístico producido por nuestros colaboradores, entre la lengua quechua y el español.

En la zona andina del Perú, las poblaciones bilingües de castellano hacen uso de dos variedades de castellano diferentes producto de la situación de contacto de lenguas entre el quechua y el castellano. Estas variedades son el castellano como segunda lengua y el castellano como lengua materna. Según Anna María Escobar (1988, p.26), la adquisición del castellano puede realizarse de manera consecutiva o simultánea con el quechua. Los bilingües que han adquirido el castellano de manera consecutiva al quechua hacen uso del castellano bilingüe (castellano como 
segunda lengua), mientras que los bilingües que han adquirido el castellano de manera simultánea con el quechua hacen uso del castellano andino (castellano como lengua materna hablado en la zona andina). En el caso de los colaboradores, ellos hacen uso del castellano bilingüe ya que durante su etapa escolar han adquirido el castellano como segunda lengua, siendo su lengua materna el quechua. Por su parte, Alberto Escobar (1978) denomina como castellano de los bilingües al «castellano de quienes, por lo común, son nativohablantes de quechua o aimara, que tienen el español como segundo idioma» (p. 29).

El interlecto, según Alberto Escobar (1978, p. 30), es una interlengua, esto es, una lengua hablada, como segunda lengua, por personas cuya lengua materna es una lengua amerindia. Es decir, se podría definir al interlecto, como un español hablado como segunda lengua o como una variedad del español que se encuentra en proceso de aprendizaje del castellano. Este es el caso de los colaboradores, ellos tienen como lengua origen al quechua y como lengua meta al castellano. En este interlecto se desarrollan las interferencias lingüística, referidas, básicamente, a situaciones de problemas o conflictos en la adquisición de una segunda lengua, resultaba adecuado para describir una situación de bilingüismo de tipo subordinado que caracterizaba el interlecto, donde se entiende que la lengua materna se constituye en un factor conflictivo para el feliz aprendizaje de la segunda lengua. Por estas razones, « el castellano de los bilingües (interlecto) que tiene el quechua como lengua materna está plagado de interferencias y que estas "parecen ser mayores en contextos pobres de aprendizaje, de escasos niveles de escolaridad» (Escobar, 1990).

\section{Metodología}

La presente investigación es de tipo descriptivo, ya que, primeramente, se describirá los conceptos más relevantes referidos al castellano bilingüe, interferencias lingüísticas, interlecto, entre otros. También, se realizará una breve descripción de la lengua materna (quechua) y de la lengua meta (castellano) con el fin de poder caracterizar las interferencias morfosintácticas de la lengua materna hacia la L2. Por otro lado, se dará una explicación a cada fenómeno lingüístico encontrado en el habla de los colaboradores.

La investigación se realizó el mes de Agosto del año 2017 en Jangas, distrito de la provincia de Huaraz en el departamento de Áncash. El corpus base del presente trabajo lo constituyen una serie de grabaciones de entrevistas realizadas a cuatro 
Interferencias morfosintácticas del quechua al castellano...

personas del sexo masculino residentes del distrito. Los hablantes se desempeñan en diferentes ocupaciones y sus edades oscilan entre los 30 y los 60 años. La tabla 1, se elaboró a fin de facilitar la mención de cada informante en su entrada correspondiente, como también, se colocaron los datos de edad, procedencia y ocupación.

Tabla 1. Datos de los colaboradores

\begin{tabular}{|l|l|l|l|l|}
\hline N. $^{\circ}$ & Nombre & Edad & Procedencia & Ocupación \\
\hline I1 & Jacinto & 32 & Jangas, Áncash & Guía turístico \\
\hline I2 & Justo Obregón & 58 & Jangas, Áncash & Alfarero \\
\hline I3 & Alberto & 60 & Jangas, Áncash & Artesano de piedra \\
\hline I4 & Heber Tafur & 40 & Jangas, Áncash & Artesano de piedra mármol \\
\hline
\end{tabular}

Para la recolección de datos, fue necesario elaborar un instrumento que permitiera recopilar el corpus; para ello, se elaboró un cuestionario con preguntas abiertas a fin de entrevistar a los colaboradores. Las preguntas giraron en torno a temas sobre sobre el trabajo que realizan, anécdotas, familia, educación y sobre el pueblo; además de información personal acerca de los entrevistados. Otro instrumento empleado fue una grabadora, con este se registró en audio toda la información que era proporcionada durante las entrevistas; las grabaciones ayudaron a la transcripción y segmentación de las frases para su posterior análisis. Asimismo, se utilizó un lápiz y un cuaderno de apuntes para escribir algunos datos relevantes que se manifestaron durante la entrevista.

Por otra parte, se consultó un número importante de libros, revistas y artículos con el fin de obtener información para el desarrollo del marco teórico y sobre los procesos que se hallan en el habla de los colaboradores.

\section{Análisis morfosintáctico}

En este apartado, se mostrarán los fenómenos lingüísticos encontrados en nuestros colaboradores como resultado de la interferencia lingüística de su lengua materna quechua al español. Los fenómenos encontrados son omisión lingüística, discordancia gramatical, inserción lingüística, pronombres anómalos, dequeísmo, loísmo, los cuales serán presentados en ese orden. 


\subsection{Omisión lingüística}

Mendoza (1991) denomina como elisión u omisión al proceso de anulación o supresión de un determinado elemento en una estructura, es decir, es un proceso mediante el cual se suprime morfemas o segmentos lingüísticos. A continuación, se mostrarán los tipos de omisiones en el español de los colaboradores.

\subsubsection{Omisión de artículos}

La carencia del determinante articular ocurre en casi todos los dialectos quechuas (Cerrón-Palomino, 2003, p. 140), ya que, en la lengua quechua, el artículo no es un elemento imprescindible para el establecimiento de la referencia, sino más bien, «existen morfemas como -qa, junto a otros enclíticos que desempeñan la función de presentadores/reconocedores y que permiten la realización efectiva de tal función: Machu-qa wañurapusqa, 'el hombre viejo se había muerto'». (Calvo, 1993, p. 42).

Por su parte, Virginia Zavala (1996) reafirma que el quechua no posee en sus sistemas la categoría de artículo, por ello suelen ser omitidos en la segunda lengua, sin embargo, «la única excepción es la palabra huk que, como un adjetivo numeral cardinal, puede también funcionar en otros contextos como un artículo indeterminado» (p. 95-96).

Como ya se mencionó, en la lengua quechua no se evidencian morfemas gramaticales equivalentes a los artículos (los, la y el), por tal razón, los colaboradores suelen omitirlos en los casos que se presentan a continuación.

\subsubsection{Omisión del artículo definido masculino los}

Con respecto a la ausencia del artículo los, Anna María Escobar (2000) afirma que «la omisión ocurre sobre todo con artículos definidos y frases nominales que están dentro de una frase preposicional [...]» (p. 38).

Ejemplos:

(1) (Los) animales también, (los) amarramos donde sea. (I-3)

(2) Yo (me) casé (a) (los) cuarenta y tres todavía. (I-4) 
Interferencias morfosintácticas del quechua al castellano...

\subsubsection{Omisión del artículo definido femenino la}

A continuación, se mostrará los casos en los que los colaboradores omiten el artículo la.

Ejemplos:

(3) Sí, (la) chacra no más acá pe, papi. (I-3)

(4) Para ahorro (de) (la) comunidad vendemos [...] (I-2)

(5) Los demás días nos dedicamos a (la) chacra. (I-3)

(6) Dentro de esta área [...] encontramos (la) cordillera. ¿Ya? (I-1)

(7) Ya, aunque sea, (la) puerta abierta deja [...] (I-2)

\subsubsection{Omisión del artículo definido masculino el}

Con respecto a los casos en los que los colaboradores omiten el artículo el. Anna María Escobar (2000, p. 39) señala que estos artículos tienden a omitirse en los sintagmas nominales de sujeto, objeto y objeto de preposición; ya que los quechuahablantes, al no tener ningún morfema que cumpla las mismas funciones que los artículos en el español, tienden a no expresarlas.

Ejemplos:

(8) Dentro de la comunidad, en (el) área norte sur encontramos [...] (I-1)

(9) Cogemos (el) martillo para esculpir mejor. (I-2)

\subsubsection{Omisión de preposiciones}

Las preposiciones pertenecen a la categoría invariable, es decir, no tienen significado propio. En palabras de Marcial Morera (1994, p. 241), sirven para relacionar un sustantivo con otro sustantivo dentro de la cadena lingüística.

Según Zavala Cisneros (1996, p. 101), el español andino se caracteriza por la omisión de preposiciones o por el empleo inadecuado de ellas; ya que la construcción nominal en la lengua quechua se encuentra formada por raíces y sufijos, los cuales se aglutinan. Es decir, en la lengua quechua no existen preposiciones que funcionen como conexión entre dos sintagmas.

Para Anna María Escobar (1988, p. 18), las preposiciones locativas y las temporales son las únicas omitidas en el habla bilingüe ya que generalmente la información que ofrece este tipo de preposiciones está contenida en el sustantivo. A continuación, se mostrará los casos en los que se evidencia esta omisión. 


\subsubsection{Omisión de la preposición a}

En algunos casos, se pudo evidenciar la omisión de la preposición 'a' ante el verbo infinitivo, tal como lo menciona Virginia Zavala (1996, p. 101), este fenómeno es el más recurrente ante dicha estructura. Además, también se evidencia la ausencia de la preposición a cuando cumple función locativa.

Ejemplos:

(10) Yo así con saco blanco, así, (a) (los) diecisiete yo (me) casé. (I-4)

(11) [...] para qué voy (a) poner(me) abrigo. (I-1)

(12) Los demás días nos dedicamos (a) (la) chacra. (I-3)

(13) Yo (me) casé (a) (los) cuarenta y tres todavía. (I-4)

(14) Desde chiquititos ellos querían ir (a) trabajar. (I-4)

\subsubsection{Omisión de la preposición de}

En el siguiente ejemplo, podemos evidenciar la omisión de la preposición de cuando cumple la función de origen o principio. Tal como lo señala Anna María Escobar (1998, p. 18), es posible la omisión de la preposición de en función de origen.

Ejemplos:

(15) Para ahorro (de) (la) comunidad vendemos [...] (I-2)

\subsubsection{Omisión de la preposición en}

En el siguiente ejemplo, se mostrará la omisión de la preposición en que cumple la función de preposición locativa. Al respecto, Anna María Escobar (1998, p.18) señala que las preposiciones locativas son las únicas omitidas en el habla bilingüe, además, es posible la omisión de la preposición en cuando cumple la función de lugar.

Ejemplos:

(16) (En) casa andamos con ropa viejo. (I-4)

\subsubsection{Omisión de pronombres}

En esta sección, se mostrará la omisión de pronombres encontrados en el habla de nuestros colaboradores, estos son los pronombres reflexivos me, te y nos. Con respecto a los pronombres reflexivos, Virginia Zavala $(1996$, p.107) señala que un 
rasgo importante del castellano andino es un uso peculiar de los pronombres reflexivos en contextos no aceptables por el español estándar. Sin embargo, en los siguientes ejemplos no se evidencia tal fenómeno, sino más bien, se muestra la omisión de estos pronombres.

\subsubsection{Omisión de pronombre reflexivo me}

Ejemplos:

(17) A (los) diecisiete yo (me) casé. (I-4)

(18) [...] para qué voy (a) poner(me) abrigo. (I-1)

(19) Yo (me) dedico acá un poco de agricultura. (I-3)

\subsubsection{Omisión de pronombre reflexivo se}

Ejemplo:

(20) La mayoría no hacen pasar a su taller porque son intermediarios, pero (se) pasan por artesanos. (I-2)

\subsubsection{Omisión de pronombre reflexivo nos}

Ejemplo:

(21) Quizá ahí también (nos) ponemos cortito, pero, no más. (I-2)

\section{Comentario:}

Con respecto al fenómeno de omisión de artículos, se puede evidenciar que estos son escasamente producidos debido a su inexistencia en el quechua. Sin embargo, en las oraciones (5), (8) y (12) podemos observar que sí se emplean los determinantes.

En los ejemplos mostrados, se evidencia la omisión de preposiciones, ya que en el quechua no existen, pues estas son expresadas como sufijo. Sin embargo, en las entradas (11), (15) y (18) se puede observar el empleo de la preposición para. Además, si bien se evidencia la omisión de la preposición de en la entrada (15), también se muestra su uso en la entrada (8). 
Por último, con respecto a los pronombres reflexivos, estos son omitidos como se muestra en la entrada (21). Sin embargo, podemos observar su empleo en la entrada (5).

\subsection{Discordancia gramatical}

En palabras de Virginia Zavala (1996, p. 46), el castellano andino presenta una cantidad inimaginable de discordancias gramaticales de género y número, ya que la lengua quechua no posee en su sistema la categoría de género.

Con respecto a la concordancia de género y número, Calvo (1993) reafirma que existen peculiaridades respecto a la falta de concordancia genérica y numérica, ya que el quechua carece de género y el pluralizador nominal tiene un comportamiento diferente al pluralizador español. Debido a que, existen «hasta cuatro tipos de pluralidad en la lengua quechua: intensivo dual (ñawi, 'los dos ojos'), intensivo global (runa, 'gente'), extensivo dual (sach'a sach'a, 'bosque') y extensivo global (pisqu-juna, 'los pájaros’)» (p. 350).

\subsubsection{Discordancia gramatical de género entre un determinante y un nombre}

Este tipo de discordancia, se muestra la falta de concordancia de género entre un determinante y un nombre. Anna María Escobar (2000, p. 77) señala que los bilingües que no aplican la regla de asignación de género del español se encuentran en una etapa intermedia de la adquisición de la segunda lengua, ya que ellos «reconocen la regla pero no conocen las excepciones».

Ejemplos:

(22) Cuando está ya la asa puesto [...]. (I-2)

(23) Sigue lloviendo y ya ha cambiado la clima. (I-1)

(24) Ese tipo trajo un piedra muy duro. (I-3)

(25) Se trabaja la asa para que quede liso. (I-2)

(26) Siempre tengo algún noticia. (I-1) 
Interferencias morfosintácticas del quechua al castellano...

\subsubsection{Discordancia gramatical de}

género entre nombre y adjetivo

En los siguientes ejemplos, se muestra la falta de concordancia de género entre el nombre y el adjetivo que lo califica.

Ejemplos:

(27) Ese tipo trajo un piedra muy duro. (I-3)

(28) [...] así falda blanco [...] (I-4)

(29) Se dedicaban puro chacra no más. (I-4)

(30) Antes era puro lana. (I-4)

(31) [... ya lo retocamos con el cuchillo, te lo forma ya la asa bonito porque se pone con barbotina todavía. (1-2)

(32) La piedra fino se trabaja mejor. (I-3)

(33) (En) casa andamos con ropa viejo. (I-4)

\subsubsection{Discordancia gramatical de número} entre el pronombre personal y el objeto

Anna María Escobar (2000, p. 57) señala que los casos de discordancia gramatical de número se dan porque una de las expresiones ya tiene el concepto de pluralidad.

Ejemplos:

(34) Yo a mis hijos le digo estudia, estudia, pero ni los tres no querían estudiar. (I-1)

\subsubsection{Discordancia gramatical de} número entre nombre y adjetivo

En esta sección, se muestra la falta de concordancia de número entre el nombre y el adjetivo que lo califica.

Ejemplos:

(35) [...] sobre nueva cosas. (I-2)

(36) En este lugar tenemos bastante instrumentos. (I-3)

(37) Los recuerdos caro eran más elaborados. (I-2) 


\section{Comentario:}

Con respecto a la discordancia de género y número, es un proceso que ocurre ya que en la lengua quechua no se evidencia la discordancia de género ni de número. Se puede evidenciar que es el fenómeno lingüístico más recurrente y el más común en el castellano andino.

Si bien el género gramatical no existe en la sintaxis quechua y, por ello, no es común encontrar frases concordantes. Cabe mencionar, que existen casos como el de la entrada (31) que sí presenta una adecuada concordancia entre el artículo y el nombre.

\subsection{Inserción lingüística}

La inserción lingüística es el proceso mediante el cual el hablante agrega o inserta elementos como preposiciones, pronombres, artículos, etc.

\subsubsection{Inserción del morfema plural -s}

En este caso, se observa la inserción del morfema plural -s al pronombre indefinido nadie. Según Enrique Pato (2013, p. 403), aunque este pronombre carece de plural, en algunas variantes del español popular andino se documenta ampliamente la forma nadies en el registro coloquial.

Ejemplo:

(38) Ya, aunque sea, (la) puerta abierta deja, regresan y nadies lleva ni nadies entra. (I-2)

Con respecto al sustantivo gentes, Virginia Zavala (1996, p. 94) menciona que estas desviaciones ocurren cuando el hablante en un enunciado también utiliza un verbo plural.

Ejemplo:

(39) Hay veces mis hijas hablan o gentes. (I-4)

\subsubsection{Inserción de morfemas diminutivos}

En el quechua, existen sufijos que se insertan a los nombres para indicar énfasis, emoción o diminutivo, dicho sufijo es -cha. Anna María Escobar (2000, p. 87) afirma que este proceso se llama «reduplicación del diminutivo» $\mathrm{y}$ «redundancia 


\section{Interferencias morfosintácticas del quechua al castellano...}

del diminutivo» ya que dentro de tal palabra o enunciado existe el significado de tamaño pequeño.

Ejemplos:

(40) Le das forma suavecito no más. (I-2)

(41) Yo también tengo recuerditos chiquititos. (I-2)

(42) Desde chiquititos ellos querían ir (a) trabajar. (I-4)

\section{Comentario:}

En este trabajo se ha considerado a la forma nadies como una inserción del morfema plural, debido a que, el adverbio de negación nadie, en su equivalente quechua, posiblemente sea flexionado en plural. La misma hipótesis se puede formular para gente, que se muestra flexionada como gentes. Sin embargo, es importante considerar que esta forma puede ser producto de la recurrente concepción colectiva de los quechuas, que se refleja en la sufijación como plural.

Con respecto a la inserción de morfemas diminutivos, este proceso se debe a que en la lengua quechua utilizan regularmente el morfema -cha, lo cual indica también un equivalente al diminutivo del español.

\subsection{Loísmo}

Según Caravedo (1990), en el español andino «se encuentra muy extendido el leísmo y ocurre con gran frecuencia el loísmo, a veces alternando en el mismo hablante o en distintos hablantes de la misma zona».

Al respecto, Lipski (1994, p. 345) señala que en el español andino bilingüe es común la falta de concordancia entre los clíticos y los objetos directos e indirectos, por ello, es habitual que los clíticos de objeto directo «lo» $\mathrm{y}$ «la» se utilicen en contextos que requieren un clítico de objeto indirecto. Este es el caso de nuestros colaboradores, los cuales utilizan el clítico «lo» en lugar de «le».

Ejemplos:

(43) [...] cuando está en estado de cuero lo damos la forma y luego lo ponemos la arcilla. (I-2)

(44) [...] esto lo pongo aquí con cuchillito y luego lo ponemos la asa para que pegue. (I-2) 


\section{Comentario:}

La falta de concordancia de clíticos es común en el castellano andino, por ello, en ambos ejemplos se puede evidenciar el uso de lo en lugar de le ante los verbos dar y poner.

\subsection{Dequeísmo}

El dequeísmo, en palabras de Fontanillo (1986), es un vicio de dicción donde se antepone de manera indebida la preposición de a la partícula que introductoria de oración completiva dependiente; generalmente este tipo de casos se presenta con verbos como decir, pensar, saber, etc.

Ejemplos:

(45) Saben de que este nevado de aquí a unos cinco años, puede desaparecer producto del descongelamiento glacial. (I-1)

(46) Muchas mineras de que eran explotadas. (I-2)

\section{Comentario:}

En ambas entradas, se evidencia el empleo de la preposición de ante una oración sustantiva. Tal como menciona el autor, podemos observar en la entrada (45) el empleo de esta partícula ante el verbo saber.

\subsection{Pronominales anómalos}

Con respecto a este fenómeno, Virginia Zavala (1999, p. 51) menciona que presenta una compleja variedad de manifestaciones, ya que en el quechua no existe una marca especial para los pronombres de objeto de tercera persona, por ello, resulta natural que los bilingües presenten diversos fenómenos desviantes.

Al respecto, Rodolfo Cerrón-Palomino (2003, p. 231), sostiene que este fenómeno podría ser un reflejo directo del sufijo aspectual quechua -rqu que connota inmediatez, urgencia e inminencia. Esto quiere decir, que mientras que para un hispanohablante esta forma puede resultar vacía, para el que la utiliza puede estar cumpliendo una función semántica que se remonta a la lengua autóctona.

Ejemplos:

(47) Las gentes de aquí se lo dedica a la comercialización por el atractivo turístico. (I-1)

(48) Casi nadies se $\mathbf{l o}$ dedica a otras cosas. (I-3) 
Interferencias morfosintácticas del quechua al castellano...

(49) El 90\% de la población se lo dedica a la ganadería. (I-3)

(50) La población deja la agricultura para dedicárselo a la ganadería (I-3)

(51) Pueden bajar pa' que lo puedan probarlo. ¿Ya? (I-1)

(52) Con el cuchillo se lo forma ya la asa bonito porque se pone con barbotina todavía. (I-2)

\section{Comentario:}

En todos los ejemplos mostrados, se evidencia que la partícula lo cumple una función vacía, sin embargo, este fenómeno podría explicarse como una partícula que surge a raíz del quechua y que busca enfatizar una función de inmediatez. Además, en las entradas (47), (48), (49), (50) y (52) se observa que este fenómeno ocurre ante la presencia del pronombre se.

\section{Conclusiones}

A partir del análisis realizado, se puede concluir lo siguiente: Las interferencias morfosintácticas que se hallan en castellano de Jangas son omisión lingüística, discordancia gramatical, inserción lingüística, loísmo, dequeísmo y pronominales anómalos. El fenómeno lingüístico con mayor marcadez es el de discordancia gramatical de género y número, este proceso ocurre porque en la lengua quechua no se evidencia la concordancia de género ni de número. Además, todos los informantes entrevistados dieron evidencias de este fenómeno. Tal y como se muestra en las entradas de la (22) a la (37). Con respecto a los pronominales anómalos, es un fenómeno que suele ser común en los colaboradores, ya que puede ser un reflejo del sufijo aspectual del quechua que connota inmediatez. Este fenómeno se muestra en las entradas (47), (48), (49), (50), (51) y (52). 


\section{Referencias bibliográficas}

Calvo J. (1993). Pragmática y gramática del quechua cuzqueño. Perú: Centro de Estudios Andinos Bartolomé de las Casas.

Caravedo, R. (1990). Sociolingüística del español de Lima. Lima: Pontificia Universidad Católica del Perú.

Cerrón-Palomino, R. (2003). Castellano andino. Aspectos sociolingüísticos, pedagógicos y gramaticales. Lima: Pontificia Universidad Católica del Perú.

Escobar, A. (1978). Variaciones sociolingüísticas del castellano en el Perú. Lima: Instituto de Estudios Peruanos.

Escobar, A. (1990). Los bilingües y el castellano en el Perú. Lima: Instituto de Estudios Peruanos.

Escobar, A. (2011). Contacto social y lingüístico. El español en contacto con el quechua en el Perú. Lima: Pontificia Universidad Católica del Perú.

Fontanillo, E. (1986). Diccionario de Lingüística. Madrid: Anaya.

Lipski, J. (1994). El español de América. Madrid: Cátedra.

Mendoza, J. (1991). Sintaxis divergente del castellano paceño. La Paz: Imprenta Papiro.

Pato, E. (2013). Nadies, plural de nadie, en español andino. Lexis, 37(2), 403-416.

Weinreich, U. (1974). Lenguas en contacto. Caracas: Universidad Central de Venezuela.

Zavala, V. (1996). El castellano de la sierra del Perú. Osaka: National Museum of Ethnology.

Zavala, V. (1999). Reconsideraciones en torno al español andino. Lexis, 23(1), 25-85. 